Check for updates

Cite this: RSC Adv., 2019, 9, 12319

Received 25th January 2019

Accepted 15th April 2019

DOI: 10.1039/c9ra00663j

rsc.li/rsc-advances

\title{
Dielectric relaxation of the double perovskite oxide $\mathrm{Ba}_{2} \mathrm{PrRuO}_{6}$
}

\begin{abstract}
Jenq-Wei Chen, (D) * Kuan Ru Chiou, An-Chih Hsueh and Ching-Ray Chang
Samples of $\mathrm{Ba}_{2} \mathrm{PrRuO}_{6}$ were prepared by the solid state reaction method and the structure was characterized by X-ray diffraction (XRD). Scanning electron microscopy (SEM) and dielectric measurements were performed in order to investigate the morphology and electric properties of the ceramics. X-ray diffraction data reveal that the $\mathrm{Ba}_{2} \mathrm{PrRuO}_{6}$ samples are of the cubic crystal structure with the space group $\mathrm{Fm} \overline{3} \mathrm{~m}$ at room temperature. The dielectric properties were studied in the range of $20 \mathrm{~Hz}$ to $1 \mathrm{MHz}$ in the temperature range from $10 \mathrm{~K}$ to $300 \mathrm{~K}$. Strong dispersion in frequency and a rapid increase in $\varepsilon^{\prime}$ are observed when $T>150 \mathrm{~K}$. The observed steps of the $\varepsilon^{\prime}(T)$ curves are correlated with the peaks of the $\tan \delta(T)$ curves, with the peak temperature shifting to higher values as the frequency increases. Impedance spectroscopy studies indicate the presence of grain and grain boundary relaxations in the sample at high temperatures, while at low temperatures only grain relaxation can be observed. Both grain and grain boundary relaxation times follow the Arrhenius law with activation energies of $0.16 \mathrm{eV}$ and $0.17 \mathrm{eV}$, respectively.
\end{abstract}

\section{Introduction}

The double perovskite oxides with the chemical formula $\mathrm{A}_{2} \mathrm{BB}^{\prime} \mathrm{O}_{6}$, where $\mathrm{A}$ is a large cation such as $\mathrm{Ba}^{2+}, \mathrm{Sr}^{2+}$, or $\mathrm{Ca}^{2+}$ and the $\mathrm{B}$ and $\mathrm{B}^{\prime}$ cations are transition metals, have attracted a lot of research activities in recent years because of their intriguing properties and technological applications in electronic devices, sensors, fuel cells, magnetic memory, capacitors and other devices. ${ }^{1-6}$ Ruthenium-containing double perovskites $\mathrm{A}_{2} \mathrm{LnRuO}_{6}$ ( $\mathrm{A}=$ alkaline earth element; $\mathrm{Ln}=$ lanthanide $)$ have been studied extensively due to their interesting structural, transport and magnetic properties. ${ }^{7-16} \mathrm{In}_{2} \mathrm{LnRuO}_{6}$, the B-site is occupied either by rare earth or transition metal ions and determines the magnetic properties. The $\mathrm{Ba}_{2} \mathrm{LnRuO}_{6}$ compounds contain an ordered arrangement of $\mathrm{Ln}^{3+} / \mathrm{Ru}^{5+}$ ions and exhibit various antiferromagnetic ordering at low temperatures. For nonmagnetic $\mathrm{Ln}^{3+}$ ions such as $\mathrm{Y}^{3+}, \mathrm{La}^{3+}$, and $\mathrm{Lu}^{3+}$, antiferromagnetic interaction is due to the ordering of the $\mathrm{Ru}^{5+}$ ions only. ${ }^{7,8,17}$ The ordering of the $\mathrm{Ru}^{5+}$ is dominated by the relative strength of the nearest neighbor $\mathrm{Ru}^{5+}$ ions through the $\mathrm{Ru}-\mathrm{O}-\mathrm{O}-\mathrm{Ru}$ pathway and the next-nearest neighbour $\mathrm{Ru}^{5+}$ ions through the $\mathrm{Ru}-\mathrm{O}-\mathrm{Ln}-\mathrm{O}-\mathrm{Ru}$ pathway. For magnetic $\mathrm{Ln}^{3+}$ ions $(\mathrm{Ln}=\mathrm{Pr}, \mathrm{Nd}$, $\mathrm{Sm}, \mathrm{Eu}, \mathrm{Tb}, \mathrm{Dy}, \mathrm{Ho}, \mathrm{Er}, \mathrm{Tm}$ and $\mathrm{Yb}$ ), long range magnetic ordering of the $\mathrm{Ln}^{3+}$ ions is also observed., ${ }^{9,13,18-21}$

The crystal and magnetic properties of $\mathrm{Ba}_{2} \mathrm{PrRuO}_{6}$ have been studied previously. ${ }^{9,22,23}$ The crystal structure of $\mathrm{Ba}_{2} \mathrm{PrRuO}_{6}$ was reported to be cubic (space group $F m \overline{3} m$ ) at room

Department of Physics, National Taiwan University, Taipei 106, Taiwan. E-mail: jwchen@phys.ntu.edu.tw temperature. ${ }^{22,24}$ Neutron diffraction study revealed the occurrence of antiferromagnetic transition with $T_{\mathrm{N}}=117 \mathrm{~K}$, with the magnetic moments of 2.2 and $2.0 \mu \mathrm{B}$ for $\mathrm{Pr}^{3+}$ ions and $\mathrm{Ru}^{5+}$ ions at low temperature, respectively. ${ }^{9}$ Although relaxation behavior has been studied previously on other complex double perovskite ceramics. ${ }^{23}$ To date, there has been no report about the ac electrical properties of $\mathrm{Ba}_{2} \mathrm{PrRuO}_{6}$ in the literature. Properties of polycrystalline ceramic materials depend on microstructure where the grain and grain boundary properties may be significantly different from each other. It is often observed that the electrical response from a material is not intrinsic and dominated by many extrinsic factors such as grain boundary, surface layers and electrode-material interface. Therefore, it is important to study the electrical properties in correlation the microstructure through grain and grain boundary analyses. In this paper, we report dielectric and impedance studies on $\mathrm{Ba}_{2} \mathrm{PrRuO}_{6}$.

\section{Experimental}

Polycrystalline $\mathrm{Ba}_{2} \mathrm{PrRuO}_{6}$ samples were prepared using the solid state reaction method. Stoichiometric amount of high purity powders of $\mathrm{BaCO}_{3}$ (99.95\%) and $\mathrm{RuO}_{2}$ (99.99\%) and $\mathrm{Pr}_{6} \mathrm{O}_{11}$ (99.99\%) were preheated at $120{ }^{\circ} \mathrm{C}$ for 8 hours and then mixed thoroughly in an agate mortar with a pestle. The mixed powder was put in the furnace and heated to $900{ }^{\circ} \mathrm{C}$ for 12 hours, with a ramping rate $5{ }^{\circ} \mathrm{C} \mathrm{min}^{-1}$. The powder was then increased to $1200{ }^{\circ} \mathrm{C}$ with an increasing rate of $2{ }^{\circ} \mathrm{C} \mathrm{min}{ }^{-1}$, and kept at that temperature for 72 hours. The powder was then 
cooled down with a cooling rate of $2{ }^{\circ} \mathrm{C} \min ^{-1}$ to $900{ }^{\circ} \mathrm{C}$ and followed by furnace cooled to room temperature.

After the heating procedure, the mixture was added with 5\% (weight percentage) polyvinyl alcohol (PVA) as the binder and reground thoroughly, pressed into disks of $1 \mathrm{~cm}$ in diameter and $1 \mathrm{~mm}$ in thickness. The disks were then sintered with the same procedure as described above. The structure of the samples were checked at room temperature with powder X-ray (XRD) using the $\mathrm{Cu}-\mathrm{K} \alpha$ radiation. Morphology and the grain size distribution of the sintered pellets were studied using a scanning electron microscope (SEM) HITCHI S-2400. Temperature-dependent dielectric measurements were performed with the measuring frequency range from $20 \mathrm{~Hz}$ to 1 MHz using HP-4284A LCR meter. Silver paste was applied on both sides of the pellet samples. The experimental temperature is between $10 \mathrm{~K}$ and $300 \mathrm{~K}$ with the sample placed in an OFHC sample holder in a closed-cycle refrigerator (CTI-Cryogenics model 22).

\section{Results and discussions}

Shown in Fig. 1(a) is the powder XRD data of the polycrystalline $\mathrm{Ba}_{2} \mathrm{PrRuO}_{6}$ sample. The XRD pattern can be indexed to the cubic crystal structure with space group $F m \overline{3} m$. The refined lattice constants are $a=b=c=0.8482 \mathrm{~nm}$. The obtained values are close to that reported. ${ }^{22,23}$ Typical SEM micrograph of the $\mathrm{Ba}_{2} \mathrm{PrRuO}_{6}$ sample is shown in Fig. 1(b). The micro-graph reveals that the sample studied consists of grains micro-meter
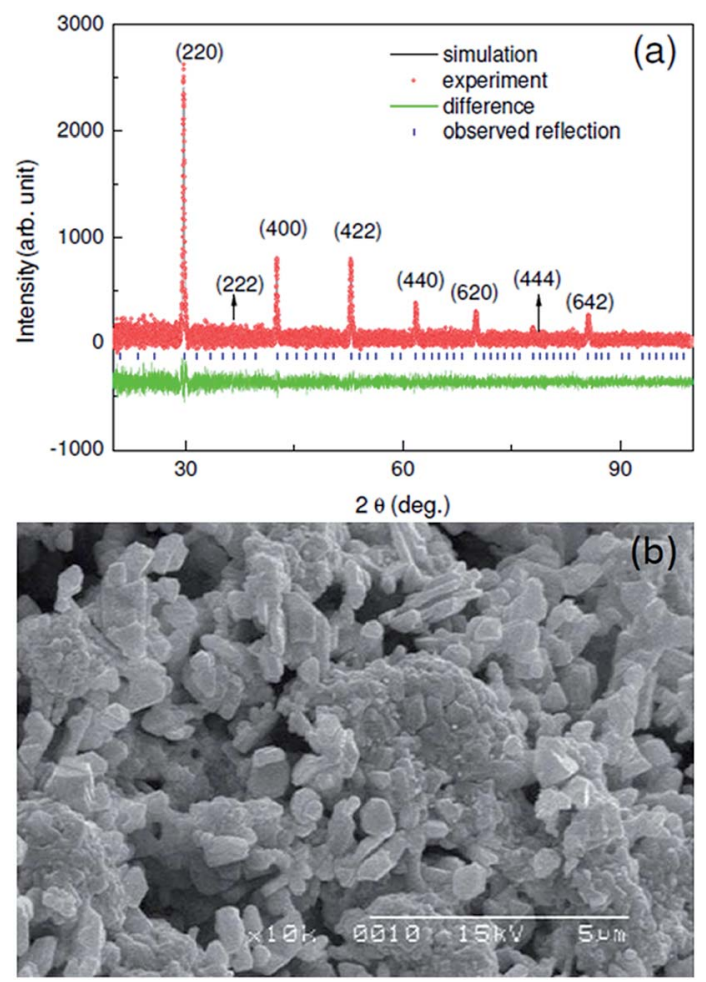

Fig. 1 (a) The $X$-ray diffraction patterns of the polycrystalline $\mathrm{Ba}_{2}$ $\mathrm{PrRuO}_{6}$ sample. (b) The SEM micrograph of the $\mathrm{Ba}_{2} \mathrm{PrRuO}_{6}$ sample. size with considerable porosity. The morphology and grain size are similar to that reported previously for $\mathrm{Ba}_{2} \mathrm{HoRuO}_{6}{ }^{25}$

The real part of dielectric permittivity $\varepsilon^{\prime}$ as a function of temperature $T$ at selected test frequency $f$ for $\mathrm{Ba}_{2} \mathrm{PrRuO}_{6}$ between $10 \mathrm{~K}$ and $300 \mathrm{~K}$ are depicted in Fig. 2(a). The $\varepsilon^{\prime}(T)$ curves exhibit distinct behaviours in different temperature regions. At the lowest temperature, $\varepsilon^{\prime}$ is almost frequency independent and approaches a value of $\sim 11$ at $10 \mathrm{~K}$. For $10 \mathrm{~K}<T<150 \mathrm{~K}, \varepsilon^{\prime}$ increases monotonically with increasing $T$ and frequency dispersion develops. Strong dispersion in frequency and rapid increase in $\varepsilon^{\prime}$ is observed for $T>150 \mathrm{~K}$. The obtained value of $\varepsilon^{\prime}$ is $\sim 2000$ at $1 \mathrm{kHz}$ at $300 \mathrm{~K}$, which decreases with increasing $f$ to a value of 50 at $1 \mathrm{MHz}$. The origin for the observed large value of the dielectric response at room temperature can be attributed to the extrinsic effect such as inhomogeneous microstructures or grain boundary effect.

Fig. 2(b) shows the real part of the dielectric permittivity $\varepsilon^{\prime} v s$. frequency $f$ of the sample at various temperatures between $10 \mathrm{~K}$ and $300 \mathrm{~K}$. As can be seen in the figure, the $\varepsilon^{\prime}(f)$ curves exhibit two distinct plateaus, separated by an inflection region, for $T>$ $180 \mathrm{~K}$. The inflection region shifts toward low frequency and then gradually disappears below $150 \mathrm{~K}$. The low frequency plateau is attributed to the grain boundary response while the high frequency dispersion is due to the grain response. The rapid increase in $\varepsilon^{\prime}$ below $200 \mathrm{~Hz}$ for $T>200 \mathrm{~K}$ is due to the electrode. Depicted in Fig. 2(c) are the loss tangent tan $\delta$ versus $T$ curves with various test frequencies $f$. The $\tan \delta$ curves exhibit a peak corresponding to the rapid increase of $\varepsilon^{\prime}(T)$. Plotted in Fig. 2(d) are the frequency dependence curves of the dielectric loss $(\tan \delta$ ) at different temperatures between $10 \mathrm{~K}$ and $300 \mathrm{~K}$. The peak temperature shifts to higher temperature as the test frequency increases.
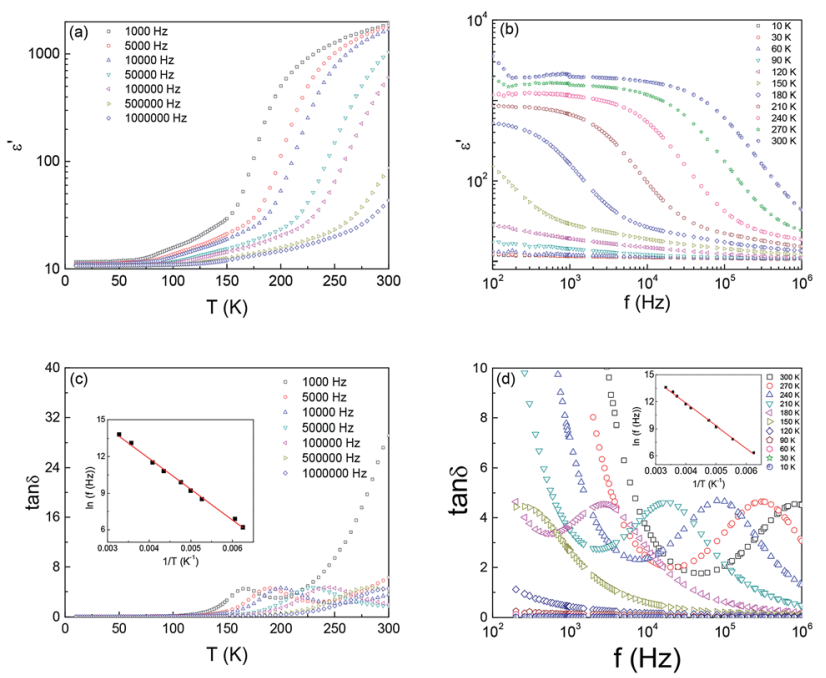

Fig. 2 (a) Real part of dielectric permittivity $\varepsilon^{\prime}$ as a function of temperature $T$ at selected test frequency $f$ for $\mathrm{Ba}_{2} \mathrm{PrRuO}_{6}$. (b) $\varepsilon^{\prime} v s$. frequency $f$ of the sample at various temperatures. (c) The loss tangent $\tan \delta$ vs. T curves with various test frequencies between $10 \mathrm{~K}$ and 300 $K$. (d) Frequency dependence of the dielectric loss ( $\tan \delta$ ) at different temperatures between $10 \mathrm{~K}$ and $300 \mathrm{~K}$. Insets of $(\mathrm{c})$ and (d): $f$ versus the reciprocal of the peak temperature $T_{\mathrm{p}}$ obtained from the $\tan \delta$ curves. 
The test frequency $f$ versus the reciprocal of the peak temperature obtained from the $\tan \delta$ curves, $T_{\mathrm{p}}$, is plotted in the insets of Fig. 2(c) and (d). It is clear that the experimental can be fitted well with the Arrhenius relation

$$
f=f_{0} \exp \left(-E_{\mathrm{a}} / k_{\mathrm{B}} T\right)
$$

where $f_{0}$ is the pre-exponential term, $E_{\mathrm{a}}$ is the activation energy, and $k_{\mathrm{B}}$ is the Boltzmann constant. The obtained fitting parameters are $E_{\mathrm{a}}=0.205 \mathrm{eV}$ and $f_{0}=1.31 \times 10^{9} \mathrm{~Hz}$.

Fig. 3(a) shows the frequency dependence of the real part of impedance $Z^{\prime}$ for various temperatures between $60 \mathrm{~K}$ and $300 \mathrm{~K}$. It shows that the value of $Z^{\prime}$ increases dramatically with decreasing $T$. It is also clear that $Z^{\prime}$ decreases with an increase in frequency, which indicates the enhancement of the ac conductivity with increasing frequency. For $T>130 \mathrm{~K}$, a temperature dependent $Z^{\prime}$ plateau from the low frequency region, followed by a negative slope at high frequency region is observed. At higher temperatures $(T>180 \mathrm{~K})$, the $Z^{\prime}(f)$ curves disclose two distinct plateaus develop at higher frequencies. The segments of nearly constant $Z^{\prime}$ become dominant as the temperature increases, suggesting the enhanced relaxation behaviour. ${ }^{26,27}$ Shown in Fig. 3(b) is the variation of the
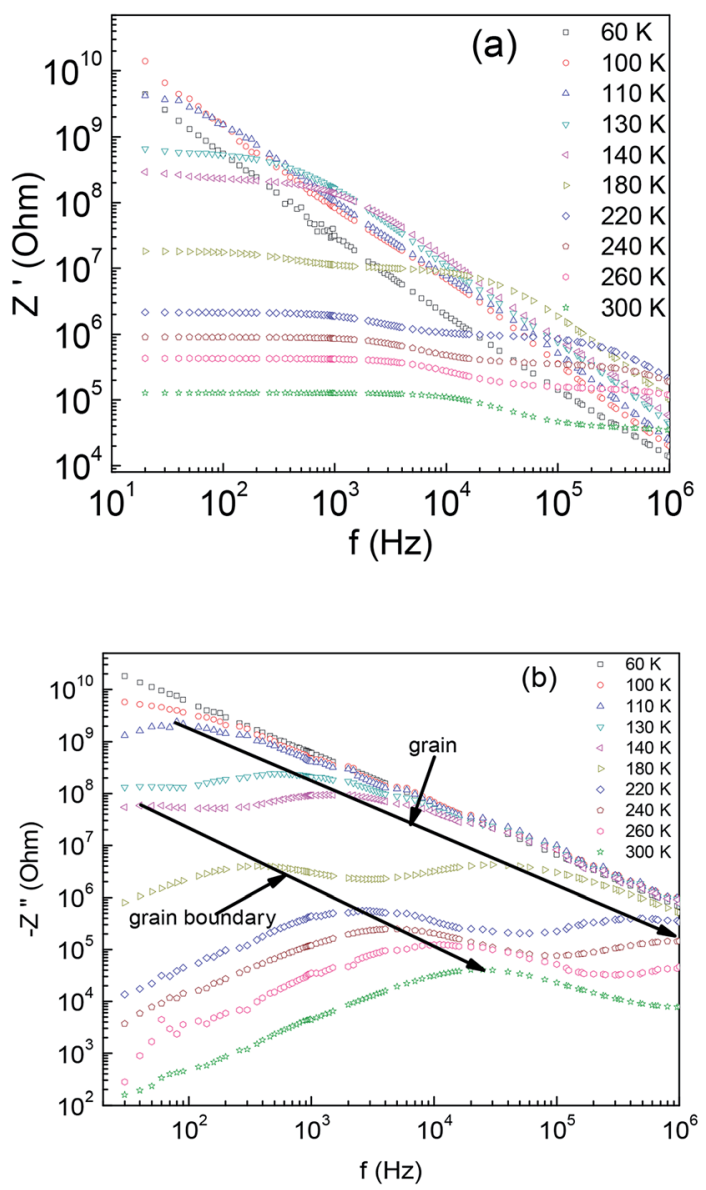

Fig. 3 The frequency dependence of (a) the real part of the impedance $Z^{\prime}$ and (b) the imaginary part of the impedance $-Z^{\prime \prime}$ of $\mathrm{Ba}_{2} \mathrm{PrRuO}_{6}$ for various temperatures between $60 \mathrm{~K}$ and $300 \mathrm{~K}$. imaginary part of impedance $Z^{\prime \prime}$ of $\mathrm{Ba}_{2} \mathrm{PrRuO}_{6}$ sample as a function of frequency for different temperatures. The $Z^{\prime \prime}(f)$ curves reveal two sets of peaks, the low frequency peaks are responsible for grain boundary contribution and the high frequency peaks corresponds to the grain response. The peak positions can be expressed as $f_{\mathrm{gb}} \sim 1 /\left(2 \pi R_{\mathrm{gb}} C_{\mathrm{gb}}\right)$ and $f_{\mathrm{g}} \sim 1 /$ $\left(2 \pi R_{\mathrm{g}} C_{\mathrm{g}}\right)$, respectively. ${ }^{28}$ With increasing temperature, the maximum shifts towards higher frequency. The Arrhenius law can be applied for both relaxations,

$$
\begin{gathered}
\tau_{\mathrm{gb}}=\tau_{\mathrm{gb} 0} \exp \left(-E_{\mathrm{gb}} / k_{\mathrm{B}} T\right), \tau_{\mathrm{gb}}=1 / 2 \pi f_{\mathrm{gb}} \\
\tau_{\mathrm{g}}=\tau_{\mathrm{g} 0} \exp \left(-E_{\mathrm{g}} / k_{\mathrm{B}} T\right), \tau_{\mathrm{g}}=1 / 2 \pi f_{\mathrm{g}}
\end{gathered}
$$

where $\tau_{\mathrm{gb} 0}$ and $\tau_{\mathrm{g} 0}$ are the characteristic times, $E_{\mathrm{gb}}$ and $E_{\mathrm{g}}$ are the activation energies for the grain boundary and grain, respectively, and $f_{\mathrm{gb}}$ and $f_{\mathrm{g}}$ are the maximum frequencies of the grain boundaries and grains. Fig. 4 shows the grain and grain boundary relaxation times $\tau_{\mathrm{g}}$ and $\tau_{\mathrm{gb}}$, respectively, obtained from the maximum frequencies versus the reciprocal of the temperature. The activation energies determined from the slopes are $E_{\mathrm{gb}} \sim 0.17 \mathrm{eV}$ for grain boundary and $E_{\mathrm{g}} \sim 0.16 \mathrm{eV}$ for the grain.

The Nyquist plots for $\mathrm{Ba}_{2} \mathrm{PrRuO}_{6}$ samples at various temperatures are shown in Fig. $5(\mathrm{a}-\mathrm{d})$. At $300 \mathrm{~K}$, only one asymmetric semicircle is observed and impedance data can be described by the Cole-Cole equation, ${ }^{29,30}$

$$
Z_{\mathrm{gb}}^{*}(\omega)=\frac{R_{\mathrm{gb}}}{1+\left(j w \tau_{\mathrm{gb}}\right)^{1-\alpha}},
$$

where $R_{\mathrm{gb}}, \tau_{\mathrm{gb}}$ and $\omega$ are the grain boundary resistance, the relaxation time for the sample and the angular frequency, respectively. The Cole-Cole parameter $\alpha$ is the measure of distribution of the relaxation time.

With decreasing temperature, the diameter of the impedance plot becomes larger and another segment at high frequency starts to develop. In the temperature range of $270-$ $180 \mathrm{~K}$ (Fig. 5(a and b)), the $Z^{*}$ plot consists of two semi-circular

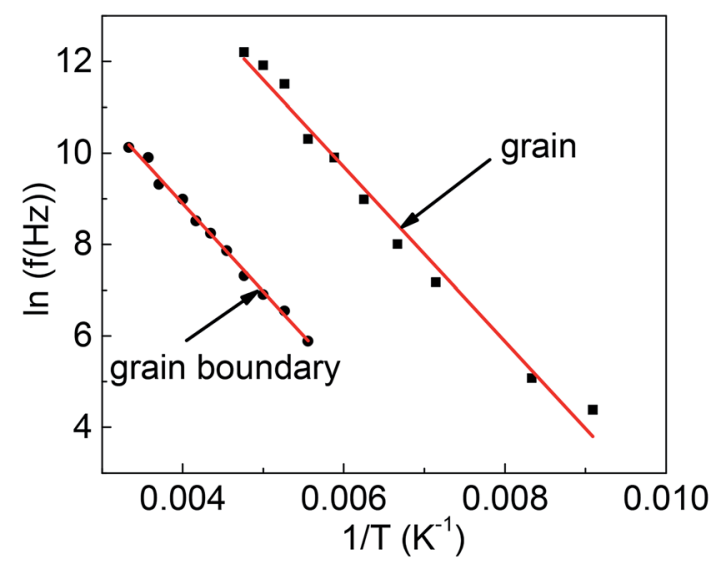

Fig. 4 The Arrhenius relation of the measuring frequency vs. $1 / T$ for grains and grain boundaries of $\mathrm{Ba}_{2} \mathrm{PrRuO}_{6}$. The solid lines are the linear fitting. 

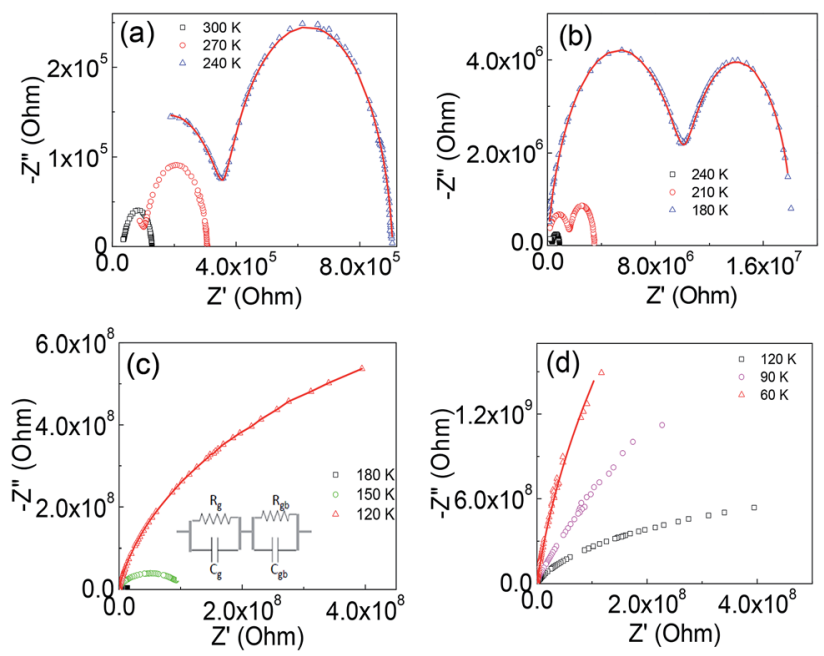

Fig. 5 (a-d) Complex plane impedance plots for $\mathrm{Ba}_{2} \mathrm{PrRuO}_{6}$ sample at selected temperatures: (a) 240, 270, $300 \mathrm{~K}$ (b) 180, 210, $240 \mathrm{~K}$ (c) 120 , $150,180 \mathrm{~K}$ (d) $60,90,120 \mathrm{~K}$. The solid lines represent the fitting from the equivalent circuits.

arcs corresponding to the grain boundary and bulk contributions. The impedance data can be analyzed in terms of two parallel $C-R$ (capacitance-resistance) circuits connected in series to represent grain and grain boundary components and $Z^{*}(\omega)=Z_{\mathrm{gb}}^{*}(\omega)+Z_{\mathrm{b}}^{*}(\omega)$. The solid lines in the figures are the fitting results. Below $180 \mathrm{~K}$, only segments of the bulk $Z_{\mathrm{b}}^{*}(\omega)$ can be observed.

For analysing the intrinsic grain dielectric relaxation, it is advantageous to display the data in terms of the imaginary part of modulus $M^{\prime \prime}$ as a function of test frequency $f$. This is because the modulus formalism $\left(M^{*}=M^{\prime}+j M^{\prime \prime}=1 / \varepsilon^{*}=j w C Z^{*}\right)$ has the advantage of eliminating the contact contributions and the relaxation with the smallest capacitance (grain) exhibits the largest peak in the $M^{\prime \prime} v$ s. $f$ plots. Depicted in Fig. 6(a) and (b) are $M^{\prime}(f)$ and $M^{\prime \prime}(f)$ curves for different temperatures between $60 \mathrm{~K}$ and $300 \mathrm{~K}$. Fig. 6(b) shows $M^{\prime \prime}(f)$ curve exhibits a peak at $\sim 360 \mathrm{~Hz}$ for $T=120 \mathrm{~K}$. And the peak position shifts to higher frequency to $\sim 480 \mathrm{kHz}$ as the temperature increases to $210 \mathrm{~K}$, and finally moves out of the measurable frequency range for $T>$ $210 \mathrm{~K}$. These peaks are related to the grain contribution and it reaches maximum at $1 /\left(2 \pi R_{\mathrm{G}} C_{\mathrm{G}}\right)$. The appearance of shoulders in the $M^{\prime \prime}$ curves are due to the grain contribution. The obtained values are $E_{\mathrm{gb}} \sim 0.175 \mathrm{eV}$ for grain boundary and $E_{\mathrm{g}} \sim 0.17 \mathrm{eV}$ for the grain. These values are close to that obtained from the $Z^{\prime \prime}(f)$ data.

According to the universal dielectric response model, ${ }^{31} \varepsilon^{\prime}$ and the measured conductivity $\sigma(f)$ at a given frequency in disordered solid can be expressed as

$$
\begin{gathered}
\varepsilon^{\prime}=\tan (s \pi / 2) \sigma_{0} f^{s-1} / \varepsilon_{0} \\
\sigma(f)=\sigma_{\mathrm{dc}}+\sigma_{\mathrm{ac}}(f)
\end{gathered}
$$

where $\sigma_{\mathrm{ac}}(f)=\varepsilon_{0} f \varepsilon^{\prime \prime}$ ( $\varepsilon_{0}$ is the permittivity of free space), is the ac conductivity which is expressed as a power law of frequency as
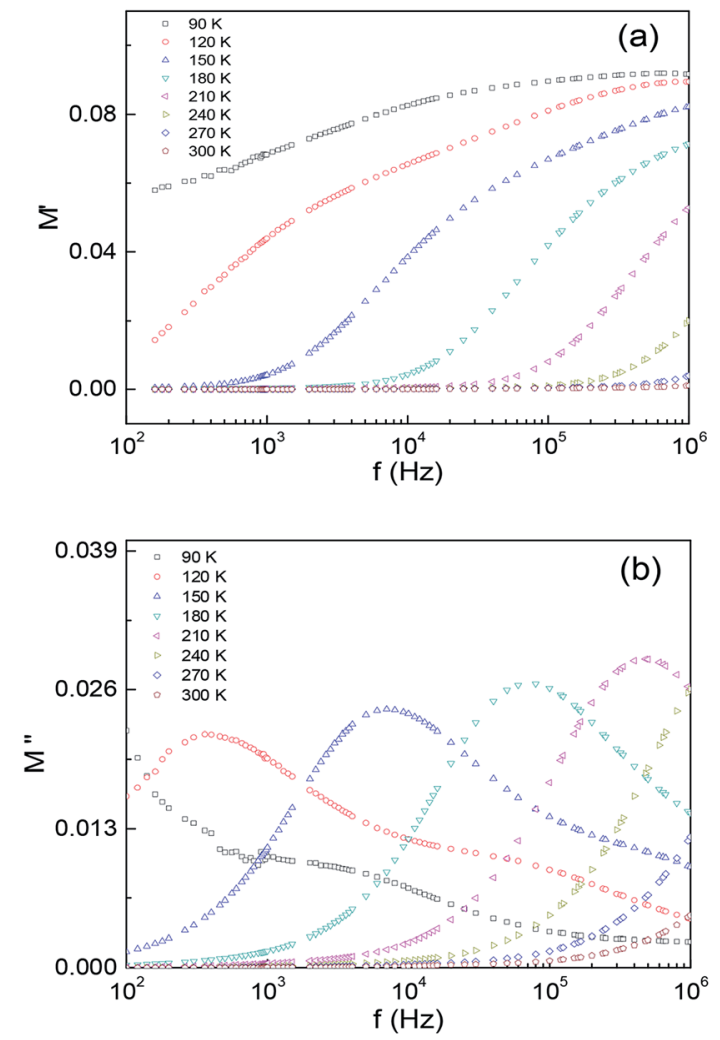

Fig. 6 The frequency dependence of (a) the real part of the electric modulus $M^{\prime}$ and (b) the imaginary part of the electric modulus $M^{\prime \prime}$ of $\mathrm{Ba}_{2} \mathrm{PrRuO}_{6}$ for various temperatures between $90 \mathrm{~K}$ and $300 \mathrm{~K}$.

$\sigma_{\text {ac }}(f)=A(T) f^{s}$, where $A(T)$ is a constant dependent on temperature, $s$ is a dimensionless parameter, and $f$ is the frequency at which the conductivity $\sigma$ was measured. The exponential increase with $T$ in loss tangent $\tan \delta$ is associated with the hopping conductivity. Which indicates that the observed relaxations may due to the hopping between the spatially fluctuating potentials. The hopping process can produce dipolar effect as well as the conductivity in the sample. This indicates that a straight line with the slope $s$ can be obtained if we plot logarithm of $\varepsilon^{\prime} f$ against logarithm of $f$ at a given temperature. Fig. 7(a) displays the $\ln \left(\varepsilon^{\prime} f\right)$ vs. $\ln (f)$ plots of $\mathrm{Ba}_{2} \mathrm{PrRuO}_{6}$ sample for a number of selected temperatures. This figure shows that two sets of straight lines can be observed in different temperature regions. At high temperatures, a straight line is observed below $\sim 100 \mathrm{kHz}$ at $300 \mathrm{~K}$, and the segment of the straight line decreases to lower frequency with decreasing temperature $T$ and finally moves below the measurement frequency. Meanwhile another set of straight lines starts to develop from the high frequency part for $T \leq 180 \mathrm{~K}$ and the segment of the lines extends to the lower frequency with decreasing $T$. Below $\sim 60 \mathrm{~K}$, a straight line in the whole test frequency region is observable. The above results suggest that the dielectric behavior at the high temperature (low frequency part) is associated with the hopping of the carriers between the grain boundaries. With decreasing temperature the localized carriers are becoming frozen and the number of hopping carriers across the grain boundary reduces 

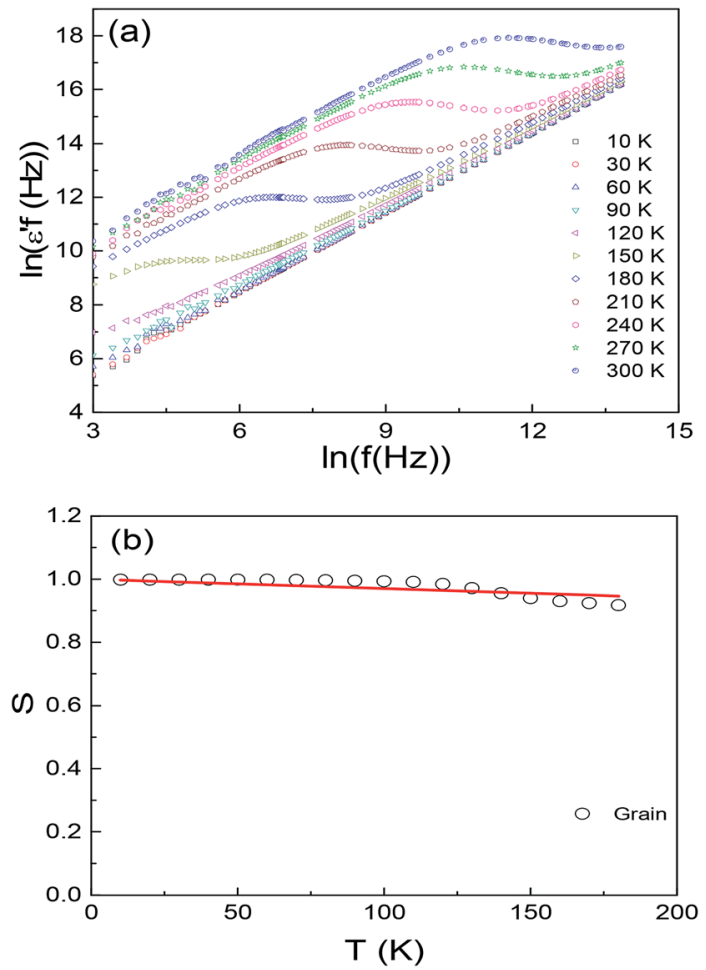

Fig. 7 (a) Variation $\ln \left(\varepsilon^{\prime} f\right)$ vs. $\ln (f)$ of $\mathrm{Ba}_{2} \mathrm{PrRuO}_{6}$ sample for selected temperatures below $300 \mathrm{~K}$, (b) temperature dependence of the frequency exponent $s$. The solid line is calculated from the $\mathrm{CBH}$ model.

dramatically. The low temperature (high frequency) dielectric behavior comes from the hopping of the carriers in the bulk. The results are in consistent with that of the impedance data. The temperature dependence of the exponent $s$ for the grain is plot in Fig. 7(b). The value of $s$ is close to 1 at $10 \mathrm{~K}$ and decreases monotonically with increasing $T$ to a value of 0.92 for $T=180 \mathrm{~K}$. Such temperature dependence of $s$ can be described with the correlated barrier hopping $(\mathrm{CBH})$ model. ${ }^{32}$

\section{Conclusions}

In summary, ceramic samples of $\mathrm{Ba}_{2} \mathrm{PrRuO}_{6}$ have been prepared by the solid state reaction method. The dielectric properties were characterized by the impedance spectroscopy. This compound exhibits a high dielectric permittivity $\left(\varepsilon^{\prime} \sim\right.$ 2000) near room temperature below $1 \mathrm{kHz}$. Impedance spectroscopy data can be modelled on equivalent circuit consisting of parallel RC elements of grain and grain boundary. The obtained values of the activation energies are $E_{\mathrm{gb}} \sim 0.17 \mathrm{eV}$ for grain boundary and $E_{\mathrm{b}} \sim 0.16 \mathrm{eV}$ for the grain.

\section{Conflicts of interest}

There are no conflicts to declare.

\section{Acknowledgements}

This work was supported by the ROC Ministry of Science and Technology under grant no. MOST 105-2112-M-002-020.

\section{Notes and references}

1 S. N. Putilin, E. V. Antipov, O. Chmaissem and M. Marezio, Nature, 1993, 362, 226.

2 S. Jin, T. H. Tiefel, M. McCormack, R. A. Fastnacht, R. Ramesh and L. H. Chen, Science, 1994, 264, 413.

3 M. K. Rath and K. T. Lee, J. Alloys Compd., 2018, 737, 152159.

4 S. W. Cheong and M. Mostovoy, Nat. Mater., 2007, 6, 13.

5 Y. H. Huang, R. I. Dass, Z. L. Xing and J. B. Goodenough, Science, 2006, 312, 254.

6 L. Troncoso, M. J. Martínez-Lope, J. A. Alonso and M. T. Fernández-Díaz, J. Appl. Phys., 2013, 113, 023511.

7 P. D. Battle, J. B. Goodenough and R. Price, J. Solid State Chem., 1983, 46, 234.

8 P. D. Battle and C. W. Jones, J. Solid State Chem., 1989, 78, 108.

9 Y. Izumiyama, Y. Doi, M. Wakeshima, Y. Hinatsu, Y. Shimojo and Y. Morii, J. Phys.: Condens. Matter, 2001, 13, 1303.

10 Y. Doi, Y. Hinatsu, A. Nakamura, Y. Ishii and Y. Morii, J. Mater. Chem., 2003, 13, 1758.

11 Y. Hinatsu, Y. Izumiyama, Y. Doi, A. Alemi, M. Wakeshima, A. Nakamura and Y. Morii, J. Solid State Chem., 2004, 177, 38.

12 H. Z. Han, H. E. Mohottala, J. I. Budnick, W. A. Hines, P. W. Klamut, B. Dabrowski and M. Maxwell, J. Phys.: Condens. Matter, 2006, 18, 2273.

13 R. Kumar, C. V. Tomy, R. Nagarajan, P. L. Paulose and S. K. Malik, Phys. B, 2009, 404, 2369.

14 R. Takahashi, R. Okazaki, Y. Yasui, I. Terasaki, T. Sudayama, H. Nakao, Y. Yamasaki, J. Okamoto, Y. Murakami and Y. Kitajima, J. Appl. Phys., 2012, 112, 073714.

15 A. A. Aczel, D. E. Bugaris, L. Li, J.-Q. Yan, C. de la Cruz, H.-C. zur Loye and S. E. Nagler, Phys. Rev. B, 2013, 87, 014435.

16 B. Ranjbar, A. Pavan, B. J. Kennedy and Z. Zhang, Dalton Trans., 2015, 44, 10689.

17 P. D. Battle and W. J. Macklin, J. Solid State Chem., 1984, 52, 138.

18 Y. Izumiyama, Y. Doi, M. Wakeshima, Y. Hinatsu, K. Oikawa, Y. Shimojo and Y. Morii, J. Mater. Chem., 2000, 10, 2364.

19 T. C. Gibb and R. Greatrex, J. Solid State Chem., 1980, 34, 279. 20 Y. Doi, Y. Hinatsu, A. Nakamura, Y. Ishii and Y. Morii, J. Mater. Chem., 2003, 13, 1758.

21 Y. Izumiyama, Y. Doi, M. Wakeshima, Y. Hinatsu, A. Nakamura and Y. Ishii, J. Solid State Chem., 2002, 169, 125. 22 P. C. Donohue and E. L. McCann, Mater. Res. Bull., 1977, 12, 519.

23 M. C. Castro Jr, C. d. A. Paschoal, F. C. Snyder and M. W. Lufaso, J. Appl. Phys., 2008, 104, 104114.

24 W. T. Fu and D. J. W. IJdo, Solid State Commun., 2005, 136, 456.

25 S. Halder, A. Dutta and T. P. Sinha, $R S C A d v ., 2017$, 7, 43812. 
26 A. Srivastava, A. Barg and F. D. Morrison, J. Appl. Phys., 2009, 105, 054103.

27 W. Chen, W. Zhu, O. K. Tan and X. F. Chen, J. Appl. Phys., 2010, 108, 034101.

28 D. C. Sinclair and A. R. West, J. Appl. Phys., 1989, 66, 3850.

29 K. S. Cole and R. H. Cole, J. Chem. Phys., 1941, 9, 342.
30 J. R. Macdonald, Impedance Spectroscopy, Wiley, New York, 1987.

31 A. K. Jonscher, Dielectric Relaxation in Solids, Chelsen, London, 1983.

32 A. Ghost, Phys. Rev. B: Condens. Matter Mater. Phys., 1990, 41, 1479. 